\title{
Recent TEM developments applied to quantum structures
}

\author{
M. Benaissa ${ }^{1}$, M. Korytov ${ }^{2}$, P.A. van Aken ${ }^{3}$ and P. Vennéguès ${ }^{2}$ \\ ${ }^{1}$ CNRST, angle Allal-FassilFAR, Madinat al-irfane, 10000 Rabat, Morocco \\ ${ }^{2}$ CNRS-CRHEA, Rue Bernard Grégory, Sophia Antipolis, 06560 Valbonne, France \\ ${ }^{3}$ Max-Planck-Institute for Intelligent Systems, Heisenbergstr-3, 70569 Stuttgart, Germany
}

\begin{abstract}
To shed light on these confined properties, a technique with a high energy-and-spatial resolution is of absolute need. Modern transmission electron microscopy (TEM) is the most suitable technique for a direct measurement of optical and structural properties at a nanometer scale. Thanks to the successful construction of aberration corrected transmission electron microscopes, the mapping of atomic positions with high accuracy becomes a routine experiment enabling therefore a more intuitive interpretation of structural deformation (strain). In addition, the recent development in energy-filters, especially when coupled to monochromated electron-beams, measurements of physical properties are achieved with unprecedented performances. The case of individual buried $\mathrm{GaN} /(\mathrm{AlGaN})$ quantum dots is discussed.
\end{abstract}

\section{INTRODUCTION}

Physical properties of quantum structures have usually been investigated using techniques with poor spatial sensitivity to explore local properties. The obtained results were then convoluted with the size distribution of the quantum structures. The case is even more complicated for buried quantum dots (QDs). In order to shed light on local physical properties, nanometer scale investigations at high spatial and energy resolutions are therefore of absolute need.

Recent development in transmission electron microscopy (TEM), coupled to a series of associated techniques, have achieved measurements of physical properties with unprecedented performances. In the present paper, our investigations will focus on two kind of technological developments, namely; aberration corrected and monochromated TEM. These two breakthroughs will be applied to directly determine, at a nanometer scale, the fluctuation of atomic and electronic structures within and around individual buried $\mathrm{GaN} /(\mathrm{AlGaN})$ QDs.

GaN/AIN QDs grown by the Stranski-Krastanow (SK) process are very promising candidates for optoelectronic device applications and therefore detailed characterization of their structure and properties has been the subject of intense investigation $[1,2]$. The threshold current density in lasers is mainly dependent on the QDs density, size and composition. However, electroluminescence is a challenging issue for AlN. The use of lower Al-content $\mathrm{Al} x \mathrm{Ga} 1-x \mathrm{~N}$ matrices is therefore an attractive alternative [3]. Although such a realization showed a great potential of $\mathrm{GaN} /(\mathrm{AlGaN})$ QDs for high-efficiency visible-light emitting devices, some fundamental issues still need further investigations, particularly the behavior of AlGaN alloy as a spacer layer in an environment where the strain-field distribution is not uniform. Therefore, a close examination of individual QDs is of central importance.

\section{EXPERIMENTAL}

The recent advent of monochromators in modern TEMs has opened new perspectives in the particular area where inelastic electron scattering are involved, usually called "low-loss regime". This part of the energy-loss spectrum is mainly dominated by volume plasmon excitations, but it also includes interband transitions. Thanks to monochromated electron beams, the energy resolution has now reached $50 \mathrm{meV}$ range [4] (at probe sizes down to few Angstroms) and thus comes close to optical spectroscopy techniques. The main advantage of the improved energy resolution in EELS is the reduced overlap of the tails of the zero-loss peak with features in the energy-loss range below $20 \mathrm{eV}$. This enables detection of so far irresolvable interband transitions and greatly simplifies quantification of band gaps and plasmon transitions.

All EELS spectra within this paper were acquired using the ZeissSESAMelectron microscope operated at an accelerating voltage of $200 \mathrm{keV}$. The microscope is equipped with an electrostatic omega-type monochromator, an in-column MANDOLINE energy-filter, and a $2 k \times 2 k C C D$ camera. All EELS spectra were obtained in STEM mode acquired at a dispersion of $0.06 \mathrm{eV} /$ channel with an incident angle of $15 \mathrm{mrad}$ and a collection angle of $10 \mathrm{mrad}$.

On the otherhand, in high-resolution transmission electron microscopy(HRTEM) the presence of spherical aberration has been, very often, exploited for producing phase contrast, which is essential for the lattice imaging of very thin objects. However, HRTEM images obtained under these conditions suffer from complicated contrast transfer and strong delocalization of higher spatial frequencies transferred by electrons scattered at larger angles. It is clear from this behavior that image artifacts due to spherical aberration are most pronounced in HRTEM images of objects involving a large number of different spatial frequencies, like quantum heterostructures. With the invention of spherical aberration correctors and its implementation into commercial TEMs [5] the situation has changed dramatically. Atomic columns in thin crystalline samples appear as bright dotson a dark background generating an image, which closely resembles the projected crystal structure. It will be shown that imaging under such conditions is able to provide the atomic scale resolution necessary 


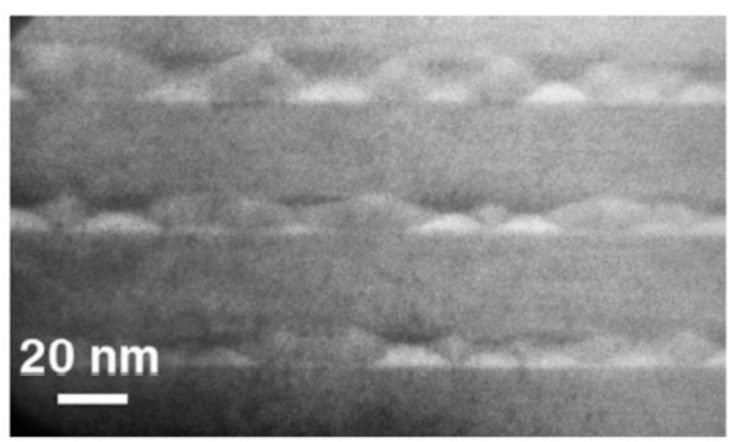

Figure 1. HAADF image of GaN QDs.

to investigate the atomic structure of very fine crystal deformation.

All our observations were done in cross-section orientation along the $\langle 1,-1,0,0\rangle$ axis. HRTEM imaging was done using Cs-corrected FEI Titan microscope $(300 \mathrm{kV})$. The local lattice parameter (LLP) inside and outside the QDs were extracted from HRTEM images using geometrical phase analysis (GPA) [6]. The reference region was chosen in the $\mathrm{A} 10.5 \mathrm{Ga} 0.5 \mathrm{~N}$ layer lying below the QD plane, where no chemical composition variations were detected.

\section{RESULTS AND DISCUSSION}

The sample architecture studied in the present paper is composed of a stack of three $\mathrm{GaN}$ QDs layers separated by a $30 \mathrm{~nm}$ Al0.5Ga0.5N spacing layer grown using molecular beam epitaxy (MBE) [7]. In order to closely examine the chemical homogeneity within our GaN/AlGaN QDs layers, high-angle annular-darkfield (HAADF) imaging with a collection angle greater than $70 \mathrm{mrad}$ was performed. In these HAADF imaging conditions, the obtained contrast is strongly dependent on the atomic number $Z$ of the existing elements; the higher the atomic number is, the brighter the corresponding contrast becomes. Whereas other perturbations, e.g., strain field, only present a marginal contribution to the imaging contrast. As can clearly be noticed from Fig. 1, GaN QDs (identified as white truncated pyramids) display a strong bright contrast while the AlGaNcap layer shows a pronounced contrast variation as a consequence of a significant chemical fluctuation.

Such behavior is noticed over the three AlGaN caplayers, particularly at the regions of AlGaN immediately on top of GaNQD surface. This AlGaN area shows a strong dark contrast compared to that of AlGaN immediately beneath the GaNQDs layer. Our result undoubtedly indicates that the $\mathrm{AlGaN}$ part above the $\mathrm{QD}$ has a significant $\mathrm{Ga}$ deficiency, or in otherwords, rich in $\mathrm{Al}$. In addition, open $\mathrm{V}$-shaped arms arising from the top rims of GaN QDs appear brighter than the rest of the $\mathrm{AlGaN}$ cap layer implying that these areas are richer in $\mathrm{Ga}$. These V-shaped arms are systematically present over the dots.

In order to further investigate this chemical composition fluctuation, or phase separation phenomenon, lowloss (or valence) EELS experiments were performed using
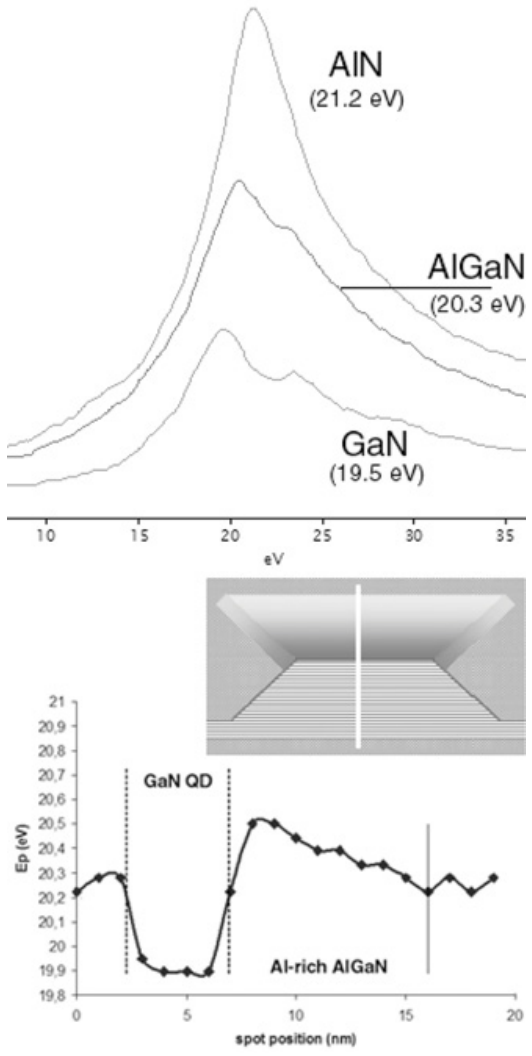

Figure 2. Valence EELS measurements.

a sub-nanometer electron probe to ensure extraction of the local information with high spatial resolution. As can be seen in Fig. 2 (top), three valence EELS spectra were first recorded from $\mathrm{GaN}$, $\mathrm{AlN}$, and $\mathrm{A} 10.5 \mathrm{Ga} 0.5 \mathrm{~N}$ thick layers.

Mainly two distinguished spectral features are revealed. The first one extends from about 19 to $22 \mathrm{eV}$, which is characteristic of unbound valence electrons performing collective oscillations; whereas the second feature extending to about $23-24 \mathrm{eV}$ is associated with the transitions between the filled $d$ bands, lying below the valence band and the empty conduction band states [8] except for AlN where such a transition is forbidden.

Using same experimental conditions, an $E p$ profile is drawn in Fig. 2 (bottom) as a function of the probe position along a line-scan as elucidated in Fig. 2 (middle). The Ep profile clearly demonstrates that $\mathrm{AlGaN}$ composition is fluctuating over the 5-nm area on top of the QD. It is worth mentioning the occurrence of a slight red shift of the energy in $E p(\mathrm{GaN}-\mathrm{QD})$ with respect to $E p(\mathrm{GaN}$-thicklayer). This increase of volume plasmon energy by $0.4 \mathrm{eV}$ is due to quantum confinement.

Besides, it is well known that the energy of volume plasmons is closely related to the valence electron density [9] which, in turn, strongly depends on the lattice parameter. In order to unambiguously reveal the origin of this Ep fluctuation, accurate measurement of local lattice parameter (LLP) were carried out using aberration corrected HRTEM (Fig. 3). Local lattice deviation (deformation) froma non-deformed reference area (taken in the A10.5Ga0.5N layer below GaN QDs) can easily be deduced. 

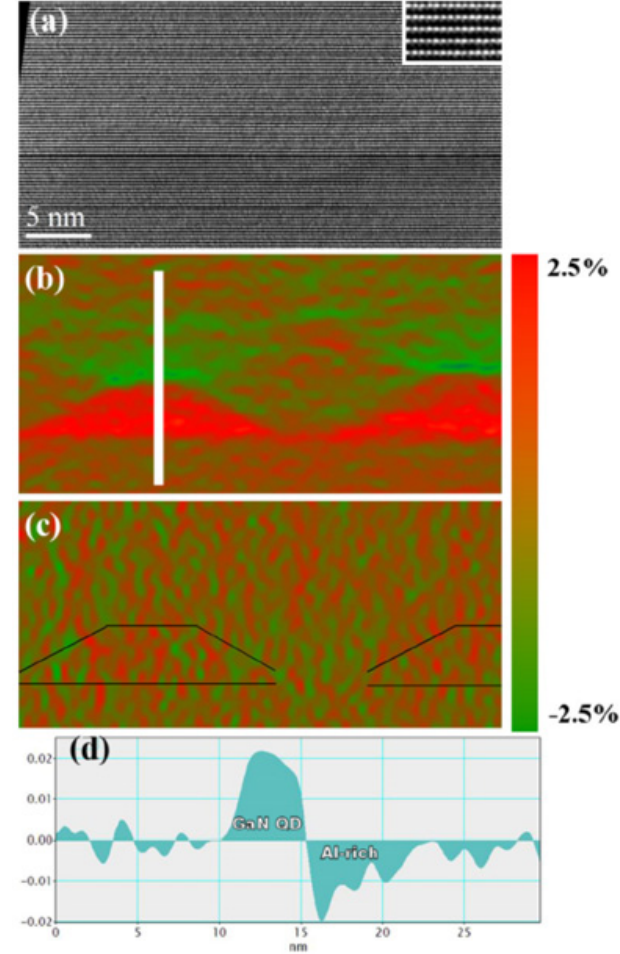

Figure 3. (color)Local lattice parameter measurements.

In other words, each distance in the HRTEM image shown in Fig. 3a is compared to the corresponding distance in the reference area (Figs. 3a-3b). This LLP deviation, or deformation $\varepsilon$, is described as:

$$
\varepsilon=(\text { LLP-LLP }- \text { reference }) / \text { LLP-reference } .
$$

In Fig. 3(b), GaN QDs and the wetting layer appear in red because the out-of-plane lattice parameters $\mathrm{d}(0002)$ of $\mathrm{GaN}$ (that is $\boldsymbol{C}$-parameter) is larger than that of the reference $\mathrm{Al} 0.5 \mathrm{Ga} 0.5 \mathrm{~N}$ layer. For the same reason, the Al-rich zones formed on top of the QDs appear in green because the out-of-plane lattice parameters d(0002) of Al-rich $\mathrm{AlGaN}$ is smaller than that of the reference Al0.5Ga0.5N. The profile (white line) traced along the growth direction (Fig. 3(d)) shows that in the Al-rich zone the LLP deviation from reference first drops down to $-2 \%$, and then gradually returns to the reference value.

At the same time, no significant deformation along the in-plane direction was measured (Fig. 3(c)). This means that the GaN QDs and the Al- and Ga-rich regions have the same in-plane lattice parameters as the $\mathrm{A} 10.5 \mathrm{Ga} 0.5 \mathrm{~N}$ reference layer which demonstrates that GaN QDs are biaxially strained. Without an aberration corrected high resolution TEM, this kind of information is simply impossible to reveal.

The origin of these compositional fluctuations in Al0.5Ga0.5N can be explained by a phase separation phenomenon provoked by the GaN QD advancing surface inhomogeneity. Indeed, two types of surface inhomogeneities are actually present inour case, namely, (i) a nonhomogeneous surface-strain distribution in addition to (ii) a nonflat overall surface. As far as the second type is concerned, theoretical studies [10] have indicated that in the case of a layer-by-layer alloy deposition on arough surface, alloy de-mixing appears to be an efficient way to partly relax the strain in the system. Second, phase separation is also thought to be a strain driven process $[11,12]$. Indeed, the surface strain distribution all over GaN QDs is expected to have a similar state as the one already reported in the case of a GaN/AIN QDs system [13], where the front surface of GaN QDs showed a modulation in the strain state inducing a modulation in the strain state distribution within the AlN barriers. In our case, an alloy such as AlGaN can behave differently, since the strain fields will direct the surface migration of $\mathrm{Ga}$ adatoms. The most attractive zones for newly deposited $\mathrm{Ga}$ adatoms correspond to the regions having an in-plane local lattice constant equal to that of unstrained GaN. Ga atoms will accumulate at the upper edges of the dots which can contribute to the increase of their lateral size [14]. It is thus expected that the produced phase separation increases the Ga concentration in the vicinity of the dots (as open V-shaped arms) at the expense of the Ga concentration in the AlGaN alloy. Therefore, the strained deposited GaN QDs provide a clearly nonhomogeneous surface strain distribution that also contributes to alloy decomposition, as it was already reported in systems such as InAs/InAlAs [15].

Finally, we attempt to combine results from aberrationcorrected-HRTEM and monochromated-VEELS results, as illustrated in Fig. 4 in order to quantify the Alrich $\mathrm{AlGaN} C$-parameter evolution as a function of Alcontent. Fig. 4a, extracted from spectra shown in Fig. 2 (top), clearly shows that plasmon energy $E p$ increases with increasing $\mathrm{Al}$ content. In Fig. $4 \mathrm{~b}$, the plasmon energy $E p$ measurement (taken from Fig. 2-bottom) is confronted with lattice deformation measurement $\varepsilon$ (taken from Fig. 3d) in order to extract their dependence. This dependence is afterwards translated into a $(E p)-(C$ parameter) dependence with the help of the dashed-line used as a calibration curve to estimate the Al-content as a function of $E p$. At this stage, we have obtained a $(E p)$-(C-parameter) dependence in addition to a $(E p)$ (Al-content) dependence. The ( $C$-parameter)-(Al-content) dependence can therefore be easily deduced. The result of these combinations is exhibited in Fig. 4c where the $C$-parameter is plotted as a function of Al-content. Interestingly, we found that the distribution of the lattice deformation obtained from aberration corrected images is directly related with the AlxGa1-xN composition deduced from monochromated loss-spectra, in accordance with the Vegard's law. The observed discrepancy is most probably due to a tensile strain which tends to reduce the $C$ parameter in the Al-rich AlGaN area as it gets closer to the GaN-QD top-face.

In conclusion, the role of combined of aberrationcorrected and monochromated TEM with emphasis on GaN/AlGaN quantum dots has been illustrated at an atomic level. As it has been demonstrated, the techniques for studying microstructural physics are 

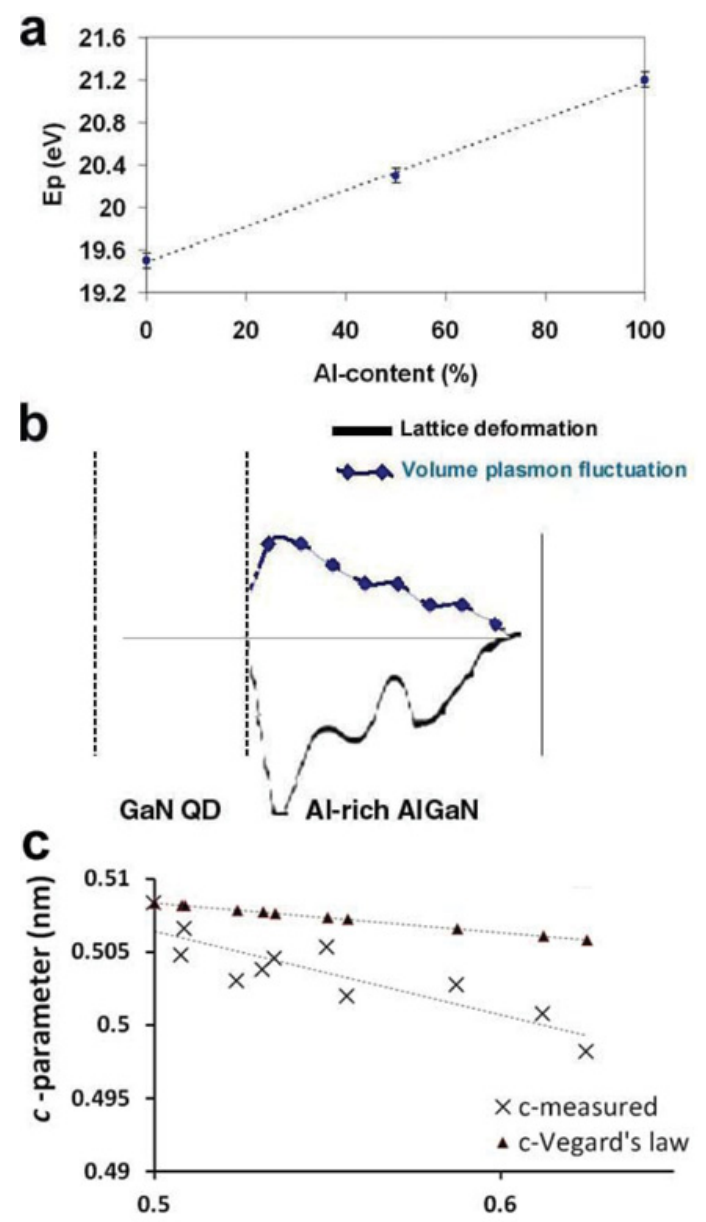

\section{Al-content (mole fraction)}

Figure 4. $C$-parameteras a function of Al-content.

sufficiently advanced and are currently a practical reality using state of the art TEM instrumentation. This should provide additional insight and aid in the understanding of nanoscale-engineered materials and help the development of new devices with superior properties.

\section{References}

[1] B. Daudin, F. Widmann, G. Feuillet, Y. Samson, M. Arlery, and J. L. Rouviere, Phys. Rev. B 56, R7069 (1997).

[2] B. Damilano, N. Grandjean, F. Semond, J. Massies, and M. Leroux, Appl. Phys. Lett. 75, 962 (1999).

[3] T. Huault, J. Brault, F. Natali, B. Damilano, D. Lefebvre, L. Nguyen, M. Leroux, and J. Massies, Appl. Phys. Lett. 92, 051911 (2008).

[4] W. Sigle, L. Gu, C. Koch, V. Srot, J. Nelayah, P. A. van Aken, Microsc Microanal 14(Suppl 2), 134 (2008).

[5] M. Haider, H. Rose, S. Uhlemann, E. Schwan, B. Kabius and K. Urban Ultramicroscopy 75, 53 (1998).

[6] M. J. Hÿ̈ch, E. Snoeck, and R. Kilaas, Ultramicroscopy 74, 131 (1998).

[7] M. Benaissa, L. Gu, M. Korytov, T. Huault, P. A. van Aken, J. Brault, and P. Vennéguès, Appl. Phys. Lett. 95, 141901 (2009).

[8] H. R. Philipp and H. Ehrenreich, Phys. Rev. 129, 1550 (1963).

[9] P. Drude, Phys. Z. 14, 161 (1900).

[10] C. Priester and G. Grenet, Phys. Rev. B 61, 16029 (2000).

[11] J. He, R. Nötzel, P. Offermans, P. M. Koenraad, Q. Gong, G. J. Hamhuis, T. J. Eijkemans, and J. H. Wolter, Appl. Phys. Lett. 85, 2771 (2004).

[12] M. V. Maximov, A. F. Tsatsul'nikov, B. V. Volovik, D. S. Sizov, Yu. M. Shernyakov, I. N. Kaiander, A. E. Zhukov, A. R. Kovsh, S. S. Mikhrin, V. M. Ustinov, Zh. I. Alferov, R. Heitz, V. A. Shchukin, N. N. Ledentsov, D. Bimberg, Yu. G. Musikhin, and W. Neumann, Phys. Rev. B 62, 16671 (2000).

[13] E. Sarigiannidou, E. Monroy, B. Daudin, J. L. Rouvière, and A. D. Andreev, Appl. Phys. Lett. 87, 203112 (2005).

[14] M. Korytov, M. Benaissa, J. Brault, T. Huault, T. Neisius, and P. Vennéguès, Appl. Phys. Lett. 94, 143105 (2009).

[15] J. Brault, M. Gendry, O. Marty, M. Pitaval, J. Olivares, G. Grenet, and G. Hollinger, Appl. Surf. Sci. 162, 584 (2000). 